Article

\title{
Simultaneous Determination of Seven Lipophilic and Hydrophilic Components in Salvia miltiorrhiza Bunge by LC-MS/MS Method and Its Application to a Transport Study in a Blood-Brain-Barrier Cell Model
}

\author{
Hui Wang 1,2, ${ }^{+}$, Mingyong Zhang ${ }^{1,2,+}$, Jiahao Fang ${ }^{1,2}$, Yuzhen He ${ }^{1,2}$, Min Liu ${ }^{3}$, Zhanying Hong ${ }^{1,2, *(D)}$ \\ and Yifeng Chai ${ }^{1,2}$ \\ 1 School of Pharmacy, Naval Medical University, Shanghai 200433, China; wanghuiuuh@163.com (H.W.); \\ myzhangchina@163.com (M.Z.); fangjiahaos@163.com (J.F.); heyuzhen9477@163.com (Y.H.); \\ yfchai@smmu.edu.cn (Y.C.) \\ 2 Shanghai Key Laboratory for Pharmaceutical Metabolite Research, Shanghai 200433, China \\ 3 Department of Pharmacy, Changhai Hospital, Naval Medical University, Shanghai 200433, China; \\ 1m_yaofen2003@163.com \\ * Correspondence: hongzhy001@163.com; Tel.: +86-218-187-1269 \\ + These authors contributed equally to this work.
}

check for updates

Citation: Wang, H.; Zhang, M.; Fang, J.; He, Y.; Liu, M.; Hong, Z.; Chai, Y. Simultaneous Determination of Seven Lipophilic and Hydrophilic Components in Salvia miltiorrhiza Bunge by LC-MS/MS Method and Its Application to a Transport Study in a Blood-Brain-Barrier Cell Model. Molecules 2022, 27, 657. https:// doi.org/10.3390/molecules27030657

Academic Editors: Monika Waksmundzka-Hajnos and Miroslaw Hawryl

Received: 16 December 2021

Accepted: 18 January 2022

Published: 20 January 2022

Publisher's Note: MDPI stays neutral with regard to jurisdictional claims in published maps and institutional affiliations.

Copyright: (C) 2022 by the authors. Licensee MDPI, Basel, Switzerland. This article is an open access article distributed under the terms and conditions of the Creative Commons Attribution (CC BY) license (https:// creativecommons.org/licenses/by/ $4.0 /)$.

\begin{abstract}
Salvia miltiorrhiza Bunge (SM) has been extensively used in Alzheimer's disease treatment, the permeability through the blood-brain barrier (BBB) determining its efficacy. However, the transport mechanism of SM components across the BBB remains to be clarified. A simple, precise, and sensitive method using LC-MS/MS was developed for simultaneous quantification of tanshinone I (TS I), dihydrotanshinone I (DTS I), tanshinone IIA (TS IIA), cryptotanshinone (CTS), protocatechuic aldehyde (PAL), protocatechuic acid (PCTA), and caffeic acid (CFA) in transport samples. The analytes were separated on a $\mathrm{C} 18$ column by gradient elution. Multiple reaction monitoring mode via electrospray ionization source was used to quantify the analytes in positive mode for TS I, DTS I, TS IIA, CTS, and negative mode for PAL, PCTA, and CFA. The linearity ranges were $0.1-8 \mathrm{ng} / \mathrm{mL}$ for TS I and DTS I, $0.2-8 \mathrm{ng} / \mathrm{mL}$ for TS IIA, 1-80 ng/mL for CTS, 20-800 ng/mL for PAL and CFA, and $10-4000 \mathrm{ng} / \mathrm{mL}$ for PCTA. The developed method was accurate and precise for the compounds. The relative matrix effect was less than $15 \%$, and the analytes were stable for analysis. The established method was successfully applied for transport experiments on a BBB cell model to evaluate the apparent permeability of the seven components.
\end{abstract}

Keywords: Salvia miltiorrhiza Bunge; LC-MS/MS; active components; transmembrane transport; BBB cell model

\section{Introduction}

Alzheimer's disease (AD) is the leading cause of dementia and one of the significant healthcare challenges of the 21st century [1]. AD is an age-related neurodegenerative disorder characterized by progressive cognitive decline and memory loss [2]. Research since the discoveries of amyloid $\beta(\mathrm{A} \beta)$ and tau protein, the main components of senile plaque (SP), and neuro-fibrillary tangles (NFT), respectively, has provided detailed information about molecular pathogenetic events of AD [3]. Additionally, oxidative stress, mitochondrial dysfunction, excessive reactive oxygen species production, lipid peroxidation, proteasomal dysfunction, microglial activation, neurotransmitter alteration, and neuroinflammation have also been implicated in AD pathology [4]. However, the cause of AD is poorly known, and there are no curative treatments despite the major expenditure of research and money over many decades [5]. Current treatment strategies only provide symptomatic relief. The common drugs, including cholinesterase inhibitors, N-methyl-D-aspartic acid (NMDA) 
receptor antagonists, and anti-inflammatory and antioxidant agents, have various adverse effects [6]. In China, traditional Chinese medicine (TCM) has a long history of treatment for $\mathrm{AD}$, and extensive progress research has been conducted for the prevention and treatment of $\mathrm{AD}$ [7].

Salvia miltiorrhiza Bunge (SM), also known as Danshen in Chinese, has been extensively used in clinics to treat stroke perimenopausal syndrome, anemia, and cardiovascular and cerebrovascular diseases for hundreds of years in China [8]. Increasing studies demonstrated that SM significantly improved the symptoms of AD and other central nervous system diseases $[9,10]$. However, the transport mechanism of SM across the blood-brain barrier (BBB) remains to be clarified. The BBB plays a vital role in maintaining the balance and stability of the brain microenvironment by maintaining restricted transport of toxic or nutrimental molecules and the removal of metabolites [11]. The BBB constitutes multiple physical and chemical barriers that restrict the movement of drugs and antigens across it, leading to minimal bioavailability of drugs in the central nervous system (CNS) [12]. As the transport mechanism of SM across the BBB has not been fully elucidated, it is necessary to establish a method to determine active components of SM for the study of transport across the BBB [13].

According to their structural characteristics, the active ingredients of SM can be classified into two groups: hydrophilic phenolic acids, such as salvianolic acid B (Sal B), salvianolic acid A (Sal A), and danshensu, protocatechuic aldehyde (PAL), protocatechuic acid (PCTA), caffeic acid (CFA), and lipophilic tanshinones, such as tanshinone I (TS I), dihydrotanshinone (DTS), tanshinone IIA (TS IIA), and cryptotanshinone (CTS) [14]. Studies have shown that both groups have multiple neuroprotective potentials relevant to $\mathrm{AD}$, such as anti-A $\beta$, antioxidant, anti-apoptotic, and anti-inflammation properties, and enhancement of cholinergic transmission [15]. High-performance liquid chromatography tandem triple quadrupole mass spectrometry has been widely used in the quantification of active components in SM [16-18]. An ultra-performance liquid chromatography tandem mass spectrometry analysis method was established and detected 12 phenolic acids and five tanshinones in SM extract solutions [16]. Liu et al. [18] established an LC-MS method for separating and detecting RA, TS I, CTS, TS IIA, and DTS I in plasma of rats after oral administration of SM. However, the samples used were plasma and brain tissue, and no research has been paid to simultaneous determination of SM components in transport samples.

This study aimed to develop an LC-MS/MS method to simultaneously determine four lipophilic tanshinones, TS I, DTS I, TS IIA, CTS, and three hydrophilic phenolic acids, PAL, PCTA, and CFA, in HBSS (Hank's Balanced Salt Solution) samples (as shown in Figure 1). Then, the LC-MS/MS method was applied for transmembrane transport study of these active components in SM on a BBB cell model.<smiles>Cc1cccc2c1ccc1occc12</smiles>

Tanshinone I<smiles>O=Cc1ccc(O)c(O)c1</smiles>

Protocatechuic aldehyde<smiles>Cc1cccc2c3c(=O)c(ccc12)C1=C(OCC1C)C3=O</smiles>

Dihydrotanshinone I

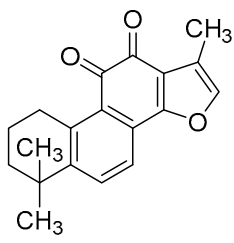

Tanshinone IIA

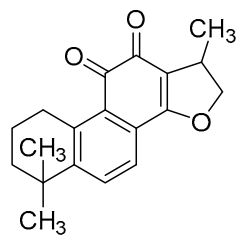

Cryptotanshinone<smiles>O=C(O)c1ccc(O)c(O)c1</smiles>

Protocatechuic acid<smiles>O=C(O)/C=C/c1ccc(O)c(O)c1</smiles>

Caffeic acid

Figure 1. Chemical structures of seven active components in Salvia miltiorrhiza Bunge. 


\section{Results and Discussion}

\subsection{Method Development}

To achieve a rapid and reliable LC-MS/MS method for determining ingredients of SM in HBSS, optimization of chromatographic separation and mass spectrometric detection parameters was systematically carried out. Seven ingredients with good stability in HBSS were selected, including water-soluble compounds PAL, PCTA, CFA, and lipid-soluble components TS IIA, CTS, TS I, and DTS I, to establish a sensitive LC-MS/MS method. In subsequent experiments, the results demonstrated that the water-soluble analytes in the negative ionization mode expressed high intensity and good sensitivity of precursor and product ions. In contrast, the lipid-soluble analytes responded well in the positive ionization mode.

For the chromatographic separation, physicochemical properties demonstrated that the seven target compounds possessed an extensive polarity range. Therefore, the gradient elution method was used to achieve high separation efficiency on the C18 column. Formic acid in water (solvent A) and acetonitrile (solvent B) were selected after optimization. A total of $0.05 \%$ formic acid was added to enhance the mass spectrometry ionization and maintain a good peak shape. Afterward, the influence of different flow rates $(0.300 \mathrm{~mL} / \mathrm{min}$, $0.400 \mathrm{~mL} / \mathrm{min}$ ) was experimentally tested. The results showed no significant differences in the retention time among these conditions, while the column pressure at $0.400 \mathrm{~mL} / \mathrm{min}$ was increased. Thus, $0.300 \mathrm{~mL} / \mathrm{min}$ was chosen as the final flow rate considering the time and cost saving. Moreover, the applicability of sulfamethoxazole (SMZ) and simvastatin was investigated as internal standards (IS). The internal standard SMZ had a good separation with the analytes and showed intense signal responses and less noise in the negative mode. At the same time, simvastatin, as a lactone compound, was very unstable in the HBSS system.

In the method described, the mobile phase consisted of water and acetonitrile using gradient elution. In the first $8 \mathrm{~min}$, all of the water-soluble analytes in the negative ionization mode, including the internal standard, were washed out in the negative mode. The lipidsoluble compounds in the positive mode were eluted after $8 \mathrm{~min}$. Therefore, ESI- scanning was used in the first $8.5 \mathrm{~min}$, and ESI + scanning was used after $8.5 \mathrm{~min}$ to avoid reducing the instrument's sensitivity due to frequent switching of positive and negative ion modes. The HBSS balanced salt, as the transport system, contains many inorganic salt ions. To protect the mass spectrometer from HBSS, the divert valve was set to direct the flow to the waste from 0 to $1.7 \mathrm{~min}$. The highlight of this study is simultaneous quantification of the seven compounds in both positive and negative ion modes with the same internal standard.

\subsection{Method Validation}

Comprehensive method validation was performed in specificity, linearity and range, accuracy and precision, matrix effect, and stability.

\subsubsection{Specificity}

Specificity was assessed by comparing the LC-MS/MS chromatogram integrity of the blank HBSS and the lowest limit of quantification (LLOQ) samples spiked with IS and the transport sample. The developed method showed no significant interfering peak from endogenous substances interferences at the retention times of seven SM components and IS in HBSS and transport samples (as shown in Figure 2). CTS had a similar retention time as the TS I, but they do not share the same fragmentation ion at the multiple reaction monitoring (MRM) mode. With the analysis of the purity of the mass chromatogram, this method can be used for the determination of seven components of SM in HBSS and transport samples. 

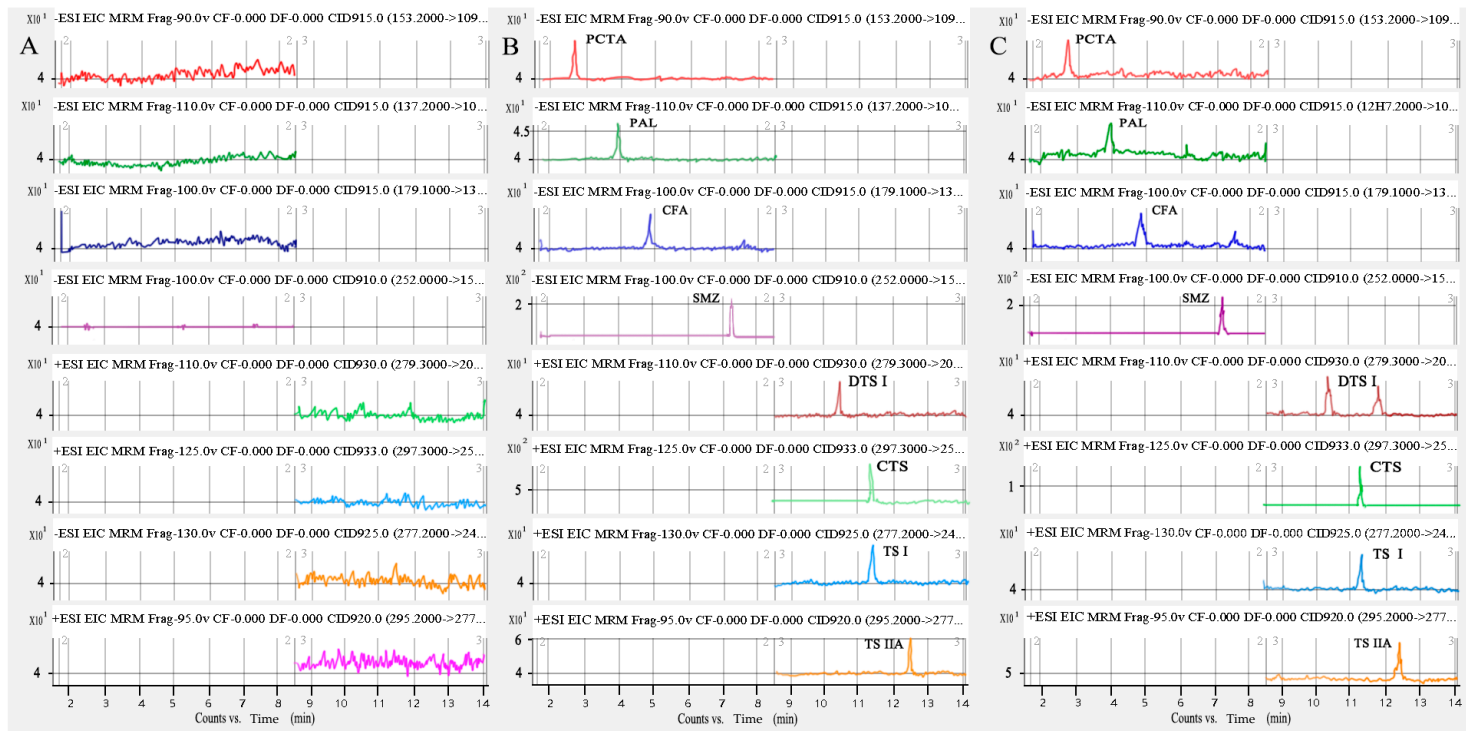

Figure 2. MRM chromatograms of seven components in Salvia miltiorrhiza Bunge and IS. (A) a blank HBSS sample; (B) a blank HBSS sample spiked with IS and seven components at LLOQ level; (C) The transport sample.

\subsubsection{Linearity and LLOQ}

Calibration standards (CS), with a concentration range of $0.1-8 \mathrm{ng} / \mathrm{mL}$ for TS I and DTS I, $0.2-8 \mathrm{ng} / \mathrm{mL}$ for TS IIA, $1-80 \mathrm{ng} / \mathrm{mL}$ for CTS, $20-800 \mathrm{ng} / \mathrm{mL}$ for PAL and CFA, and $10-4000 \mathrm{ng} / \mathrm{mL}$ for PCTA, were prepared and analyzed in duplicate in three separate analytical runs. Linear regression analysis was performed and correlation coefficients $\left(\mathrm{r}^{2}\right)$ for calibration generated were 0.98 for CTS, and were greater than 0.99 for other six components in HBSS, indicating good linearity over the range studied for the seven components of SM. The regression equations, correlation coefficients, and linear ranges of seven components of SM are shown in Table 1.

Table 1. Calibration curves of the seven components of Salvia miltiorrhiza Bunge in HBSS.

\begin{tabular}{cccccc}
\hline Analytes & Calibration Curve & Weighting & Linear Range (ng/mL) & $\mathbf{r}^{2}$ & LLOQ (ng/mL) \\
\hline TS I & $\mathrm{Y}=0.1068 X+0.0102$ & $1 / \mathrm{X}^{2}$ & $0.1-8$ & 0.9934 & 0.1 \\
DTS I & $\mathrm{Y}=0.2497 \mathrm{X}+0.0043$ & $1 / \mathrm{X}$ & $0.1-8$ & 0.9988 & 0.1 \\
TS IIA & $\mathrm{Y}=0.8962 X+0.0426$ & $1 / \mathrm{X}^{2}$ & $0.2-8$ & 0.9922 & 0.2 \\
CTS & $\mathrm{Y}=0.2353 \mathrm{X}+0.0537$ & $1 / \mathrm{X}^{2}$ & $1-80$ & 0.9837 & 1 \\
PAL & $\mathrm{Y}=0.0024 \mathrm{X}+0.0092$ & $1 / \mathrm{X}$ & $20-800$ & 0.9965 & 20 \\
PCTA & $\mathrm{Y}=0.0124 \mathrm{X}+0.0451$ & $1 / \mathrm{X}^{2}$ & $10-4000$ & 0.9965 & 10 \\
CFA & $\mathrm{Y}=0.0195 \mathrm{X}+0.0250$ & $1 / \mathrm{X}$ & $20-800$ & 0.9989 & 20 \\
\hline
\end{tabular}

\subsubsection{Precision and Accuracy}

The intra-day precision and accuracy were evaluated by analyzing five replicate $\mathrm{QC}$ samples at three levels, low QC (LQC), medium QC (MQC), and high QC (HQC). The inter-day precision and accuracy were determined by running three validation batches on each of three consecutive days. As shown in Table 2, the intra-day and inter-day precision and accuracy of seven components at high, medium, and low concentrations were within the acceptable range, except for the intra-day accuracy of CFA at high concentrations. The CFA's intra-day accuracy of the highest concentration on the first day was $81.51 \%$, but its intra-day accuracy was within the acceptable range. For the six components of SM except for CFA, the precision of LOQ of each component was not exceeding $20 \%$, and the accuracy of LOQ of each compound was within $80-120 \%$. Therefore, this method was considered to be accurate and precise. 
Table 2. Accuracy and precision of seven constituents of Salvia miltiorrhiza Bunge in HBSS.

\begin{tabular}{|c|c|c|c|c|c|c|c|c|c|c|c|c|c|}
\hline \multirow{2}{*}{ Analytes } & \multirow{2}{*}{$\underset{\text { (ng/mL) }}{\text { Con. }}$} & \multicolumn{3}{|c|}{ Validation Run 1} & \multicolumn{3}{|c|}{ Validation Run 2} & \multicolumn{3}{|c|}{ Validation Run 3} & \multicolumn{3}{|c|}{ Between-Run } \\
\hline & & $\underset{(\mathrm{ng} / \mathrm{mL})}{\operatorname{Mean} \pm S D}$ & Accuracy (\%) & $\begin{array}{c}\text { RSD } \\
(\%)\end{array}$ & $\underset{(\mathrm{ng} / \mathrm{mL})}{\operatorname{Mean} \pm \mathrm{SD}}$ & Accuracy (\%) & $\underset{(\%)}{\text { RSD }}$ & $\underset{(\mathrm{ng} / \mathrm{mL})}{\operatorname{Mean} \pm \mathrm{SD}}$ & Accuracy (\%) & $\underset{(\%)}{\text { RSD }}$ & $\underset{(\mathrm{ng} / \mathrm{mL})}{\operatorname{Mean} \pm \mathrm{SD}}$ & Accuracy (\%) & $\begin{array}{c}\text { RSD } \\
(\%)\end{array}$ \\
\hline \multirow{3}{*}{ TS I } & 0.2 & $0.17 \pm 0.02$ & $86.46 \pm 9.86$ & 9.83 & $0.22 \pm 0.01$ & $107.58 \pm 6.93$ & 5.77 & $0.21 \pm 0.01$ & $104.97 \pm 8.08$ & 6.87 & $0.20 \pm 0.03$ & $99.67 \pm 12.44$ & 12.88 \\
\hline & 1 & $1.00 \pm 0.03$ & $100.21 \pm 2.91$ & 2.59 & $1.13 \pm 0.03$ & $113.05 \pm 2.91$ & 2.67 & $1.09 \pm 0.06$ & $108.61 \pm 6.61$ & 5.44 & $1.07 \pm 0.07$ & $107.29 \pm 6.97$ & 6.49 \\
\hline & 8 & $7.52 \pm 0.24$ & $94.06 \pm 3.34$ & 3.17 & $7.61 \pm 0.25$ & $95.10 \pm 3.54$ & 3.32 & $8.16 \pm 0.56$ & $102.06 \pm 8.10$ & 6.87 & $7.74 \pm 0.47$ & $96.72 \pm 5.91$ & 6.10 \\
\hline \multirow[b]{2}{*}{ TS IIA } & 0.4 & $0.34 \pm 0.01$ & $85.18 \pm 3.95$ & 4.25 & $0.42 \pm 0.01$ & $104.73 \pm 4.04$ & 3.46 & $0.35 \pm 0.01$ & $87.88 \pm 3.55$ & 3.51 & $0.37 \pm 0.04$ & $92.93 \pm 9.86$ & 10.44 \\
\hline & 2 & $1.90 \pm 0.07$ & $95.14 \pm 4.08$ & 3.82 & $2.13 \pm 0.09$ & $106.48 \pm 5.15$ & 4.34 & $2.07 \pm 0.04$ & $103.54 \pm 2.12$ & 1.93 & $2.03 \pm 0.12$ & $101.72 \pm 6.19$ & 6.09 \\
\hline \multirow{3}{*}{ DTS I } & 0.2 & $0.18 \pm 0.01$ & $87.28 \pm 4.63$ & 4.45 & $0.20 \pm 0.01$ & $102.02 \pm 5.19$ & 4.54 & $0.18 \pm 0.02$ & 91.9013 .54 & 13.16 & $0.19 \pm 0.02$ & $93.73 \pm 10.34$ & 10.86 \\
\hline & 1 & $0.97 \pm 0.03$ & $97.16 \pm 3.27$ & 3.01 & $1.07 \pm 0.04$ & 107.054 .69 & 3.93 & $0.93 \pm 0.04$ & $93.10 \pm 4.91$ & 4.72 & $0.99 \pm 0.07$ & $99.10 \pm 7.28$ & 7.35 \\
\hline & 8 & $7.14 \pm 0.21$ & $89.29 \pm 2.88$ & 2.88 & $7.91 \pm 0.93$ & $98.89 \pm 13.01$ & 11.75 & $6.81 \pm 0.51$ & $85.17 \pm 7.11$ & 7.47 & $7.29 \pm 0.80$ & $91.12 \pm 10.03$ & 11.00 \\
\hline \multirow{3}{*}{ CTS } & 2 & $1.83 \pm 0.05$ & $91.60 \pm 2.26$ & 2.46 & $2.03 \pm 0.06$ & $101.61 \pm 3.48$ & 3.06 & $2.23 \pm 0.05$ & $111.61 \pm 3.18$ & 2.45 & $2.03 \pm 0.17$ & $100.89 \pm 8.74$ & 8.54 \\
\hline & 20 & $17.73 \pm 0.32$ & $88.67 \pm 1.77$ & 1.80 & $19.00 \pm 0.67$ & $94.99 \pm 3.75$ & 3.53 & $22.26 \pm 0.54$ & $111.30 \pm 3.04$ & 2.44 & $19.66 \pm 2.05$ & $98.32 \pm 10.24$ & 10.43 \\
\hline & 80 & $69.54 \pm 1.78$ & $86.92 \pm 2.49$ & 2.55 & $71.83 \pm 1.22$ & $89.78 \pm 1.77$ & 1.70 & $70.83 \pm 1.39$ & $88.53 \pm 1.97$ & 1.96 & $70.65 \pm 1.83$ & $99.31 \pm 2.29$ & 2.58 \\
\hline \multirow{2}{*}{ PAL } & 40 & $39.61 \pm 6.39$ & $99.01 \pm 3.53$ & 3.09 & $40.43 \pm 1.56$ & $101.09 \pm 4.53$ & 3.87 & $45.40 \pm 1.28$ & $113.50 \pm 3.58$ & 2.82 & $42.09 \pm 3.09$ & $105.22 \pm 7.72$ & 7.33 \\
\hline & 800 & $811.61 \pm 12.22$ & $101.46 \pm 1.71$ & 1.51 & $808.07 \pm 4.92$ & $101.02 \pm 0.70$ & 0.61 & $778.96 \pm 53.75$ & $98.63 \pm 7.73$ & 6.81 & $803.87 \pm 32.47$ & $100.49 \pm 1.05$ & 4.04 \\
\hline \multirow{3}{*}{ PCTA } & 20 & $22.87 \pm 0.76$ & $113.70 \pm 3.79$ & 3.31 & $18.30 \pm 1.35$ & $91.43 \pm 4.78$ & 7.37 & $18.34 \pm 0.57$ & $91.70 \pm 3.18$ & 3.12 & $19.62 \pm 2.36$ & $97.89 \pm 11.01$ & 12.01 \\
\hline & 200 & $228.23 \pm 9.87$ & $109.51 \pm 4.96$ & 1.26 & $204.31 \pm 2.58$ & $102.16 \pm 1.44$ & 1.26 & $206.55 \pm 7.03$ & $103.30 \pm 3.93$ & 3.40 & $213.03 \pm 13.40$ & $104.99 \pm 4.82$ & 6.29 \\
\hline & 4000 & $3710.42 \pm 288.72$ & $92.76 \pm 8.07$ & 7.78 & $3556.74 \pm 44.97$ & $88.91 \pm 1.25$ & 1.26 & $3688.29 \pm 34.90$ & $92.20 \pm 0.99$ & 0.95 & $3651.82 \pm 189.36$ & $91.29 \pm 4.74$ & 5.19 \\
\hline \multirow{3}{*}{ CFA } & 40 & $37.74 \pm 1.59$ & $94.66 \pm 4.04$ & 4.22 & $39.00 \pm 0.78$ & $97.48 \pm 2.19$ & 2.01 & $39.63 \pm 2.31$ & $99.07 \pm 6.45$ & 5.83 & $38.87 \pm 1.92$ & $97.07 \pm 4.64$ & 4.94 \\
\hline & 200 & $194.22 \pm 6.35$ & $97.12 \pm 3.53$ & 3.27 & $201.53 \pm 13.94$ & $100.78 \pm 7.81$ & 6.92 & $193.81 \pm 10.40$ & $96.90 \pm 5.83$ & 5.37 & $196.52 \pm 11.66$ & $98.27 \pm 5.84$ & 5.93 \\
\hline & 800 & $652.11 \pm 4.53$ & $81.51 \pm 0.64$ & 0.69 & $728.80 \pm 30.19$ & $91.10 \pm 4.37$ & 4.14 & $695.55 \pm 5.20$ & $86.92 \pm 0.78$ & 0.75 & $688.53 \pm 39.42$ & $86.06 \pm 4.93$ & 5.73 \\
\hline
\end{tabular}




\subsubsection{Matrix Effect}

Matrix effects of seven compounds of SM at low, medium, and high QC are shown in Table 3, respectively. The matrix effect of the target compound was similar at LQC, MQC, and HQC concentrations, all of which were enhanced or inhibited. Furthermore, for seven compounds, the RSD of the internal standard normalized matrix factor from the six batches appeared to be less than $15 \%$ at both tested concentration levels, thus meeting the acceptance criteria. Therefore, seven compounds of SM can be accurately quantified in positive and negative ion modes by the same IS in this research.

Table 3. Matrix effect of seven constituents of Salvia miltiorrhiza Bunge at three different concentrations in HBSS.

\begin{tabular}{ccccccc}
\hline \multirow{2}{*}{ Analytes } & \multicolumn{2}{c}{ LQC } & \multicolumn{2}{c}{ MQC } & \multicolumn{2}{c}{ HQC } \\
\cline { 2 - 7 } & Mean \pm SD (\%) & RSD (\%) & Mean \pm SD (\%) & RSD (\%) & Mean \pm SD (\%) & RSD (\%) \\
\hline TS I & $94.33 \pm 4.46$ & 4.73 & $99.28 \pm 2.78$ & 2.8 & $95.33 \pm 1.86$ & 1.95 \\
DTS I & $87.76 \pm 3.89$ & 4.43 & $88.34 \pm 5.36$ & 6.07 & $86.91 \pm 3.96$ & 4.55 \\
TS IIA & $86.30 \pm 5.53$ & 6.41 & $96.86 \pm 6.81$ & 7.03 & $89.81 \pm 6.29$ & 7.01 \\
CTS & $88.11 \pm 7.13$ & 8.09 & $91.26 \pm 8.03$ & 8.8 & $86.45 \pm 5.86$ & 6.78 \\
PAL & $106.06 \pm 6.03$ & 5.69 & $116.50 \pm 14.41$ & 12.37 & $118.96 \pm 10.00$ & 8.41 \\
PCTA & $101.00 \pm 1.00$ & 0.99 & $108.48 \pm 14.50$ & 13.36 & $106.06 \pm 5.76$ & 5.43 \\
CFA & $99.97 \pm 9.51$ & 9.51 & $103.06 \pm 6.82$ & 6.61 & $104.63 \pm 6.77$ & 6.47 \\
\hline
\end{tabular}

\subsubsection{Stability}

Although the samples were processed immediately, stability of the analytes at autosampler for $24 \mathrm{~h}$ and at $-80^{\circ} \mathrm{C}$ for 15 days was also assessed in the current study to extend the application. As shown in Table 4, the RSD of stability of each component was less than $15 \%$, indicating good stability of seven compounds in HBSS. The good stabilities of the components ensured the veracity of the quantitation results by this method.

Table 4. Stability of seven constituents of Salvia miltiorrhiza Bunge at three different concentrations in HBSS.

\begin{tabular}{|c|c|c|c|c|c|}
\hline \multirow{2}{*}{ Analytes } & \multirow{2}{*}{ Concentration (ng/mL) } & \multicolumn{2}{|c|}{ Autosampler (24 h) } & \multicolumn{2}{|c|}{ Long Term $\left(-80^{\circ} \mathrm{C}, 15\right.$ Days $)$} \\
\hline & & Mean \pm SD (\%) & RSD (\%) & Mean \pm SD (\%) & RSD (\%) \\
\hline \multirow{3}{*}{ TS I } & 0.2 & $104.83 \pm 12.38$ & 11.81 & $112.64 \pm 2.51$ & 2.23 \\
\hline & 1 & $94.45 \pm 5.86$ & 6.2 & $111.60 \pm 4.40$ & 3.94 \\
\hline & 8 & $93.83 \pm 3.75$ & 4 & $110.22 \pm 9.21$ & 8.36 \\
\hline \multirow{3}{*}{ DTS I } & 0.2 & $88.70 \pm 3.67$ & 4.14 & $113.43 \pm 10.52$ & 9.27 \\
\hline & 1 & $91.52 \pm 3.06$ & 3.34 & $114.08 \pm 2.21$ & 1.94 \\
\hline & 8 & $89.13 \pm 2.15$ & 2.41 & $99.99 \pm 6.54$ & 6.54 \\
\hline \multirow{3}{*}{ TS IIA } & 0.4 & $100.64 \pm 14.49$ & 14.39 & $108.42 \pm 15.37$ & 14.17 \\
\hline & 2 & $99.36 \pm 13.77$ & 13.86 & $113.91 \pm 1.68$ & 1.47 \\
\hline & 8 & $93.08 \pm 11.14$ & 11.97 & $109.50 \pm 5.61$ & 5.12 \\
\hline \multirow{3}{*}{ CTS } & 2 & $107.58 \pm 4.68$ & 4.35 & $110.59 \pm 4.75$ & 4.3 \\
\hline & 20 & $94.78 \pm 3.77$ & 3.97 & $110.62 \pm 4.01$ & 3.63 \\
\hline & 80 & $94.90 \pm 3.73$ & 3.93 & $91.81 \pm 5.50$ & 5.99 \\
\hline \multirow{3}{*}{ PAL } & 40 & $88.42 \pm 5.31$ & 6.01 & $106.24 \pm 5.99$ & 5.64 \\
\hline & 200 & $102.92 \pm 11.45$ & 11.12 & $101.76 \pm 8.94$ & 8.78 \\
\hline & 800 & $101.06 \pm 1.83$ & 1.81 & $92.28 \pm 4.85$ & 5.25 \\
\hline \multirow{3}{*}{ PCTA } & 20 & $96.14 \pm 4.10$ & 4.27 & $114.06 \pm 2.31$ & 2.03 \\
\hline & 200 & $102.10 \pm 6.02$ & 5.9 & $110.63 \pm 1.60$ & 1.45 \\
\hline & 4000 & $91.74 \pm 2.51$ & 2.74 & $91.53 \pm 5.04$ & 5.5 \\
\hline \multirow{3}{*}{ CFA } & 40 & $85.7 \pm 0.42$ & 0.5 & $113.78 \pm 1.90$ & 1.67 \\
\hline & 200 & $91.08 \pm 8.57$ & 9.41 & $111.44 \pm 1.95$ & 1.75 \\
\hline & 800 & $91.58 \pm 6.17$ & 6.74 & $106.34 \pm 6.10$ & 5.74 \\
\hline
\end{tabular}




\subsection{BBB Cell Model Transport Study Application}

The validated LC-MS/MS method was applied to evaluate the permeability of the seven components of SM in a BBB cell model. The trans-epithelial electric resistance (TEER) reached the maximum value of $33.04 \pm 5.02 \Omega \cdot \mathrm{cm}^{2}$ two days after hBMEC cells were seeded on the apical side of the transwell, which is close to the reported value, and meets the requirements of the in vitro BBB model [19]. By measuring the concentration of SM components in the samples obtained from the transport experiment, the apparent permeability coefficient $\left(\mathrm{P}_{\mathrm{app}}\right)$ and efflux rate (ER) cumulative transport was calculated, and the results are shown in Table 5. On the BBB cell model, within $120 \mathrm{~min}$, the $\mathrm{P}_{\text {app (AP-BL) }}$ of CFA, PAL, and PCTA was greater than $1 \times 10^{-6} \mathrm{~cm} / \mathrm{s}$, indicating that they were easily absorbed in the BBB model. The $P_{\text {app (AP-BL) }}$ of DTS I and CTS were greater than $1 \times 10^{-7} \mathrm{~cm} / \mathrm{s}$ and less than $1 \times 10^{-6} \mathrm{~cm} / \mathrm{s}$, indicating moderate absorption in the model. TS IIA's $P_{\text {app (AP-BL) }}$ was less than $1 \times 10^{-7} \mathrm{~cm} / \mathrm{s}$, indicating it was difficult to be absorbed in the model [20]. Generally speaking, when the ER is greater than 2.0, it may be considered a substrate of the efflux transporter, and when the ER is less than 0.5 , the compound is actively taken up $[21,22]$.

Table 5. Apparent permeability coefficient and efflux ratio of Salvia miltiorrhiza Bunge components in BBB cell model (Mean $\pm \mathrm{SD}, n=3$ ).

\begin{tabular}{cccc}
\hline Analytes & $\begin{array}{c}\mathbf{P}_{\mathbf{a p p}} \mathbf{( A P - B L )} \\
\mathbf{c m} / \mathbf{s}\end{array}$ & $\begin{array}{c}\mathbf{P}_{\mathbf{a p p}(\mathbf{B L}-A P)} \\
\mathbf{c m} / \mathbf{s}\end{array}$ & ER \\
\hline TS IIA & $(3.757 \pm 1.723) \times 10^{-8}$ & $(2.528 \pm 0.773) \times 10^{-8}$ & 0.673 \\
DTS I & $(4.643 \pm 2.012) \times 10^{-6}$ & $(3.617 \pm 1.082) \times 10^{-6}$ & 0.779 \\
CFA & $(2.147 \pm 1.010) \times 10^{-5}$ & $(2.252 \pm 0.954) \times 10^{-5}$ & 1.049 \\
CTS & $(4.977 \pm 1.587) \times 10^{-6}$ & $(5.125 \pm 1.584) \times 10^{-6}$ & 1.030 \\
PAL & $(1.516 \pm 0.179) \times 10^{-5}$ & $(1.220 \pm 0.021) \times 10^{-5}$ & 0.805 \\
PCTA & $(4.369 \pm 1.410) \times 10^{-5}$ & $(4.132 \pm 0.288) \times 10^{-5}$ & 0.946 \\
\hline
\end{tabular}

The ER values of TS IIA, DTS I, CFA, CTS, PAL, and PCTA were 0.673, 0.779, 1.049, $1.030,0.805$, and 0.946 , respectively, indicating that the absorption of the above compounds was close to their efflux rates in the BBB cell model. These results suggest that the transport mode of these compounds in the BBB cell model established in this study may be passive diffusion [23]. Treatment with TS IIA was found to suppress disruption of the BBB [24], and also polarized transport of CTS was found with facilitated efflux from the abluminal side to luminal side in endothelial cell monolayers [25], which are consistent with our results and would be scientific evidence to support the application of the components of SM to a certain extent.

\section{Materials and Methods}

\subsection{Chemicals}

TS I, DTS I, TS IIA, CTS, PAL, PCTA, and CFA reference standards were purchased from Shanghai Standard Technology Co., Ltd. (Shanghai, China). SMZ, used as the internal standard, was supplied by the National Institutes for Food and Drug Control (Beijing, China). Hank's Balanced Salt Solution (HBSS) was supplied by Gibco (Thermo Scientific, Waltham, MA, USA). Acetonitrile, methanol, and formic acid (HPLC grade) were purchased from CNW (Shanghai, China). Dimethyl sulfoxide (DMSO) was supplied by Thermo Scientific (Waltham, MA, USA). Water was prepared from the Merck ultra-pure water apparatus. Other reagents were of analytical grade.

\subsection{Cell Culture}

Endothelial cell medium (ECM), penicillin, fetal calf serum, trypsin, streptomycin, HBSS, and other culture reagents were purchased from Gibco (Carlsbad, CA, USA). Human brain microvascular endothelial cells (hBMECs) were purchased from ScienCell (Carlsbad, 
CA, USA). 12-well transwell plates with polystyrene inserts $(0.4 \mu \mathrm{m}$ pore size and $12 \mathrm{~mm}$ in diameter) were obtained from Corning Costar (Cambridge, MA, USA). The epithelial voltammeter was obtained from Electrical Resistance System (Millicell ERS-2, Canton, MA, USA). hBMECs cells were maintained in ECM supplemented with $10 \%$ fetal bovine serum (FBS),100 units $/ \mathrm{mL}$ penicillin and $100 \mathrm{mg} / \mathrm{mL}$ streptomycin in $5 \% \mathrm{CO}_{2}$ at $37^{\circ} \mathrm{C}$.

\subsection{Instrumentation and Conditions}

Quantitative analysis of the seven components was performed by liquid chromatographytriple quadrupole tandem mass spectrometry (LC-MS/MS), which consisted of an HPLC system (Agilent 1260 Infinity, Santa Clara, CA, USA) coupled with a triple quadrupole mass spectrometer with an electrospray ionization interface (Agilent 6460, Santa Clara, CA, USA).

Chromatographic separation was performed using an Agilent poroshell 120 EC-C18 column $(3.0 \mathrm{~mm} \times 100 \mathrm{~mm}, 2.7 \mu \mathrm{m})$ maintained at $25^{\circ} \mathrm{C}$. The flow rate was $0.3 \mathrm{~mL} / \mathrm{min}$, and the volume of injection was $5 \mu \mathrm{L}$. The mobile phase consisted of $0.05 \%$ formic acid in an aqueous solution as solvent $\mathrm{A}$ and acetonitrile solution as solvent $\mathrm{B}$. The column was eluted with a gradient of $85-50 \% \mathrm{~A}$ at $0-3.5 \mathrm{~min}, 50-10 \% \mathrm{~A}$ at $3.5-6 \mathrm{~min}, 10-5 \% \mathrm{~A}$ at 6-7.5 $\mathrm{min}$, and $5 \% \mathrm{~A}$ at 7.5-14 $\mathrm{min}$.

Mass spectrometry quantitative detection was operated in positive mode and negative mode. The MS parameters were as follows: capillary voltage of $4.0 \mathrm{kV}(\mathrm{ESI}+) / 3.5 \mathrm{kV}$ (ESI-), fragmentor voltage of $120.0 \mathrm{~V}$, drying gas flow of $11.0 \mathrm{~L} / \mathrm{min}$, and a gas temperature of $350{ }^{\circ} \mathrm{C}$. The MRM mode was employed to quantify PAL, PCTA, CFA, and SMZ in negative ion mode in the first $8.5 \mathrm{~min}$, and TS I, TS IIA, DTS I, and CTS in positive ion mode after $8.5 \mathrm{~min}$. The related parameters were listed in Table 6.

Table 6. The optimized mass spectrometric parameters of seven components in Salvia miltiorrhiza Bunge and IS.

\begin{tabular}{|c|c|c|c|c|c|c|c|}
\hline Analytes & $\begin{array}{l}\text { Precursor } \\
\quad(m / z)\end{array}$ & $\begin{array}{l}\text { Product } \\
(\mathrm{m} / \mathrm{z})\end{array}$ & $\begin{array}{l}\text { Frag. } \\
\text { (V) }\end{array}$ & $\begin{array}{l}\text { CE } \\
(\mathrm{eV})\end{array}$ & Dwell & Cell Accelerator Voltage & Polarity \\
\hline TS I & 277.2 & 249.2 & 130 & 25 & 100 & 8 & Positive \\
\hline DTS I & 279.3 & 204.9 & 110 & 30 & 100 & 7 & Positive \\
\hline TS IIA & 295.2 & 277.2 & 95 & 20 & 100 & 7 & Positive \\
\hline CTS & 297.3 & 251.4 & 125 & 33 & 100 & 7 & Positive \\
\hline PAL & 137.2 & 108.2 & 110 & 30 & 100 & 7 & Negative \\
\hline PCTA & 153.2 & 109.1 & 90 & 15 & 100 & 5 & Negative \\
\hline CFA & 179.1 & 135.1 & 100 & 15 & 100 & 7 & Negative \\
\hline SMZ (IS) & 252.0 & 156.1 & 100 & 10 & 100 & 5 & Negative \\
\hline
\end{tabular}

\subsection{Stock Solutions, Calibration Solutions, and Quality Control Samples}

Stock solutions of the TS I, TS IIA, DTS I, and CTS were prepared by dissolving each accurately weighed standard in DMSO and further diluting with methanol to obtain a $10 \mu \mathrm{g} / \mathrm{mL}$ final concentration. Stock solutions of the PAL, PCTA, CFA, and SMZ (IS) were prepared by dissolving each accurately weighed standard in methanol to obtain a $1 \mathrm{mg} / \mathrm{mL}$ final concentration.

The mixed working solution for calibration standard containing $100 \mathrm{ng} / \mathrm{mL}$ of TS I, DTS I and TS IIA, $1000 \mathrm{ng} / \mathrm{mL}$ of CTS, $10 \mu \mathrm{g} / \mathrm{mL}$ of PAL and CFA, and $50 \mu \mathrm{g} / \mathrm{mL}$ PCTA was prepared by diluting the stock solutions in methanol, and further diluted to a series of concentrations with methanol. Working solutions for QC samples at three levels were obtained using different preparations of stock solutions by diluting analytes in methanol. The concentrations of seven reference compounds in QC working solutions were as follows: 2, 10, and $80 \mathrm{ng} / \mathrm{mL}$ for TS I; 2, 10, and $80 \mathrm{ng} / \mathrm{mL}$ for DTS I; 4, 20, and $80 \mathrm{ng} / \mathrm{mL}$ for TS IIA; 20, 200, and $800 \mathrm{ng} / \mathrm{mL}$ for CTS; 400, 2000, and $8000 \mathrm{ng} / \mathrm{mL}$ for PAL; 200, 2000, and $4000 \mathrm{ng} / \mathrm{mL}$ for PCTA; and 400, 2000, and $8000 \mathrm{ng} / \mathrm{mL}$ for CFA. The internal standard (IS) working solution was obtained by diluting the IS stock solutions in methanol, yielding 
a $200 \mathrm{ng} / \mathrm{mL}$ solution. All working solutions for CS and QC were stored at $-80{ }^{\circ} \mathrm{C}$. CS samples and QC samples were prepared by mixing $10 \mu \mathrm{L}$ of working solution with $90 \mu \mathrm{L}$ of HBSS, respectively.

\subsection{Sample Preparation}

A $20 \mu \mathrm{L}$ of IS working solution was added to $100 \mu \mathrm{L}$ of CS or QC samples, and samples were mixed and centrifuged at $4{ }^{\circ} \mathrm{C}, 12,000 \mathrm{rpm}$ for $15 \mathrm{~min}$. The supernatant was transferred to autosampler vials with inserts before analysis. A volume of $5 \mu \mathrm{L}$ of the supernatant was injected into the LC-MS/MS system.

\subsection{Validation Procedure}

A total of six different batches of blanks were spiked at the LLOQ level and were processed to assess the selectivity of the assay. LC-MS/MS chromatograms of the blanks and LLOQ samples and the transport samples were monitored and compared for chromatographic integrity and potential interferences. The seven analytes in spiked samples should be identifiable, discrete, and reproducible.

Linearity and range were investigated by constructing a calibration curve based on a series of concentrations. The calibration curve was constructed by plotting the peak area ratios (y) of analytes to IS versus the concentrations of the analytes, using weighted linear least-squares regression. The LLOQ was defined as the concentration producing $\mathrm{S} / \mathrm{N}$ ratio of 10 . The calibration curves were acceptable if $75 \%$ of all non-zero CS were within $\pm 15 \%$ of the nominal concentrations or $\pm 20 \%$ of the lower limit of quantification.

A total of five replicates of $\mathrm{LQC}, \mathrm{MQC}, \mathrm{HQC}$ were analyzed in three analytical runs. The accuracy and precision were expressed as relative errors (RE) and relative standard deviation (RSD), respectively. The mean value of accuracy should be within $\pm 15 \%$ of the nominal value. The precision determined at each concentration level should be an RSD less than $15 \%$.

The matrix effect was evaluated by comparing peak areas of spiked samples (the peak area in HBSS) to the mixed standard solution (the peak area in a neat solution) at high, medium, and low concentrations. The internal standard normalized matrix effect was calculated.

Stability was evaluated by analyzing LQC, MQC, and HQC concentrations at autosampler for $24 \mathrm{~h}$ and $-80^{\circ} \mathrm{C}$ for 15 days with five replicates. Stability was expressed in terms of accuracy (RE) and coefficient of variation (RSD). The samples were considered stable if accuracy (RE) was within $\pm 15 \%$ of the nominal values and precision (RSD) was less than $15 \%$.

\subsection{Transport Study in a BBB Cell Model}

The hBMECs cells were seeded at a density of $3 \times 10^{5}$ cells per well on the transwell plates in EMC. Apical side volumes were $0.5 \mathrm{~mL}$, and the medium was changed every day. Basal side volumes were $1.5 \mathrm{~mL}$, and the medium was changed every other day. The medium was changed until confluent monolayers were formed. The integrity and transport capacity of the hBMEC cell monolayer was checked by measuring the TEER using an epithelial voltammeter, following the protocols suggested by the manufacturer. TEER provides information on ion current resistance across cell monolayers related to the integrity of tight junctions between cells. When the TEER reached the maximum value, the cell monolayer was used for the transport assay.

The permeability experiments were carried out bi-directionally, with working solutions containing one test component, TSI $(1.25 \mu \mathrm{g} / \mathrm{mL})$, DTSI $(0.15 \mu \mathrm{g} / \mathrm{mL})$, CTS $(1.25 \mu \mathrm{g} / \mathrm{mL})$, CFA $(10 \mu \mathrm{g} / \mathrm{mL})$, PAL $(10 \mu \mathrm{g} / \mathrm{mL})$, and PCTA $(20 \mu \mathrm{g} / \mathrm{mL})$. Aliquots of the donor compartment were collected after several time points $(15,30,60$, and $120 \mathrm{~min})$ and replaced with drug-free assay buffer. The transwell plates were incubated on an orbital shaker at $37^{\circ} \mathrm{C}$ for the entire experimental time. All experiments were performed in triplicate, and withdrawn samples were stored in $-80{ }^{\circ} \mathrm{C}$ fridge before LC-MS/MS analysis. 
For the BBB cell model, the apparent permeability coefficient, $\mathrm{P}_{\mathrm{app}}$ in apical-tobasolateral (AP-BL) or basolateral-to-apical (BL-AP) direction, and efflux rate of each component were subsequently calculated according to the following equation:

$$
\mathrm{P}_{\mathrm{app}}=\{\mathrm{dQ} / \mathrm{dt}\} \times\left\{1 /\left(\mathrm{A} \times \mathrm{C}_{0}\right)\right\},
$$

where $\mathrm{Q}$ is the accumulation quantity of the compound in the receiver side $(\mu \mathrm{g}), \mathrm{dQ} / \mathrm{dt}$ is the rate of appearance of the compound in the receiver side $(\mu \mathrm{g} / \mathrm{s}), \mathrm{C}_{0}$ is the initial concentration in the donor side $(\mu \mathrm{g})$, and A is the surface area of the membrane insert $\left(\mathrm{cm}^{2}\right)$. Furthermore, efflux ratios (ER) were calculated according to the following equation:

$$
\mathrm{ER}=\mathrm{P}_{\mathrm{app}(\mathrm{BL}-\mathrm{AP})} / \mathrm{P}_{\mathrm{app}}(\mathrm{AP}-\mathrm{BL}),
$$

where $\mathrm{P}_{\mathrm{app}}$ (BL-AP) and $\mathrm{P}_{\mathrm{app}}$ (AP-BL) are the $\mathrm{P}_{\mathrm{app}}$ values in the direction basolateral-to-apical and apical-to-basolateral, respectively.

\section{Conclusions}

This study developed a simple, precise, and sensitive LC-MS/MS method to simultaneously determine TS I, DTS I, TS IIA, CTS, PAL, CFA, and PCTA in HBSS samples. A total of seven compounds in positive and negative ion modes can be accurately quantified with the same IS. The method was well validated in terms of specificity, linearity, LLOQ, accuracy, precision, matrix effect, and stability, and the results met the FDA's requirements of drug quantitative analysis. The established method was successfully applied for transmembrane transport experiments to determine seven target components on a BBB cell model. This is the first study that simultaneously analyzed TS I, DTS I, TS IIA, CTS, PAL, CFA, and PCTA in HBSS samples. The application of transmembrane transport study on a BBB cell model revealed the absorption of the above compounds was close to their efflux rates in the BBB cell model, indicating that the transport mode of these compounds in the BBB cell model may be passive diffusion. The results provided a pre-clinical insight into the interaction between SM components and transporters on the BBB, which would be scientific evidence to support the better application of SM in treating $\mathrm{AD}$ to a certain extent.

Author Contributions: Conceptualization, H.W. and M.Z.; methodology, M.Z.; software, H.W.; validation, M.L., Y.H. and J.F.; data curation, H.W.; writing —original draft preparation, H.W. and M.Z.; writing—review and editing, Z.H.; supervision, Z.H. and Y.C.; project administration, Z.H.; funding acquisition, Z.H. All authors have read and agreed to the published version of the manuscript.

Funding: This research was funded by National Natural Science Foundation of China, grant number 81872829 and 81673386.

Institutional Review Board Statement: Not applicable.

Informed Consent Statement: Not applicable.

Conflicts of Interest: The authors declare no conflict of interest.

Sample Availability: Samples of the compounds TS I, DTS I, TS IIA, CTS, PAL, PCTA, and CFA are not available from the authors.

\section{References}

1. Tiwari, S.; Atluri, V.; Kaushik, A.; Yndart, A.; Nair, M. Alzheimer's disease: Pathogenesis, diagnostics, and therapeutics. Int. J. Nanomed. 2019, 14, 5541-5554. [CrossRef] [PubMed]

2. Paraskevaidi, M.; Allsop, D.; Karim, S.; Martin, F.L.; Crean, S. Diagnostic Biomarkers for Alzheimer's Disease Using Non-Invasive Specimens. J. Clin. Med. 2020, 9, 1673. [CrossRef]

3. Polis, B.; Samson, A.O. Role of the metabolism of branched-chain amino acids in the development of Alzheimer's disease and other metabolic disorders. Neural Regen. Res. 2020, 15, 1460-1470. [CrossRef]

4. Tonnies, E.; Trushina, E. Oxidative Stress, Synaptic Dysfunction, and Alzheimer's Disease. J. Alzheimer's Dis. 2017, 57, 1105-1121. [CrossRef] 
5. Gupta, G.L.; Samant, N.P. Current druggable targets for therapeutic control of Alzheimer's disease. Contemp. Clin. Trials 2021, 109, 106549-106560. [CrossRef]

6. Soheili, M.; Karimian, M.; Hamidi, G.; Salami, M. Alzheimer's disease treatment: The share of herbal medicines. Iran. J. Basic Med. Sci. 2021, 24, 123-135.

7. Howes, M.J.R.; Fang, R.; Houghton, P.J. Effect of Chinese Herbal Medicine on Alzheimer's Disease. Int. Rev. Neurobiol. 2017, 135, 29-56.

8. Su, C.Y.; Ming, Q.L.; Rahman, K.; Han, T.; Qin, L.P. Salvia miltiorrhiza: Traditional medicinal uses, chemistry, and pharmacology. Chin. J. Nat. Med. 2015, 13, 163-182. [CrossRef]

9. Guo, Y.; Dong, X.; Zhang, R.; Zhong, Y.; Yang, P.; Zhang, S.Y. Salvia miltiorrhiza improves Alzheimer's disease: A protocol for systematic review and meta-analysis. Medicine 2020, 99, e21924-e21928. [CrossRef]

10. Zhang, M.; Liu, Y.; Liu, M.; Liu, B.; Li, N.; Dong, X.; Hong, Z.; Chai, Y. UHPLC-QTOF/MS-based metabolomics investigation for the protective mechanism of Danshen in Alzheimer's disease cell model induced by A $\beta_{1-42}$. Metabolomics 2019, 15, 13. [CrossRef]

11. Bagchi, S.; Chhibber, T.; Lahooti, B.; Verma, A.; Borse, V.; Jayant, R.D. In-vitro blood-brain barrier models for drug screening and permeation studies: An overview. Drug Des. Dev. Ther. 2019, 13, 3591-3605. [CrossRef]

12. Harilal, S.; Jose, J.; Parambi, D.G.T.; Kumar, R.; Unnikrishnan, M.K.; Uddin, M.S.; Mathew, G.E.; Pratap, R.; Marathakam, A.; Mathew, B. Revisiting the blood-brain barrier: A hard nut to crack in the transportation of drug molecules. Brain Res. Bull. 2020, 160, 121-140. [CrossRef]

13. Yu, P.F.; Wang, W.Y.; Eerdun, G.; Wang, T.; Zhang, L.M.; Li, C.; Fu, F.H. The Role of P-Glycoprotein in Transport of Danshensu across the Blood-Brain Barrier. Evid.-Based Complement. Altern. Med. 2011, 2011, 713523. [CrossRef] [PubMed]

14. Li, Y.Y.; Hou, Z.N.; Su, F.; Chen, J.P.; Zhang, X.D.; Xu, L.; Yang, D.F.; Liang, Z.S. Quantitative Determination and Validation of Four Ketones in Salvia miltiorrhiza Bunge Using Quantitative Proton Nuclear Magnetic Resonance Spectroscopy. Molecules 2020, 25, 2043. [CrossRef]

15. Zhang, X.Z.; Qian, S.S.; Zhang, Y.J.; Wang, R.Q. Salvia miltiorrhiza: A source for anti-Alzheimer's disease drugs. Pharm. Biol. 2016, 54, 18-24. [CrossRef]

16. Zhang, X.; Yu, Y.; Cen, Y.; Yang, D.; Qi, Z.; Hou, Z.; Han, S.; Cai, Z.; Liu, K. Bivariate Correlation Analysis of the Chemometric Profiles of Chinese Wild Salvia miltiorrhiza Based on UPLC-Qqq-MS and Antioxidant Activities. Molecules 2018, $23,538$. [CrossRef]

17. Xie, Q.; Yuan, H.; Liu, Y.; Qiu, Y.; Jian, Y.; Li, B.; Peng, C.; Zhou, S.; Yang, C.; Wang, W. Simultaneous Determination of 19 Bioactive Constituents in QishenYiqi Dropping Pills by Ultra-Performance Liquid Chromatography Coupled with Triple Quadrupole Mass Spectrometry. J. AOAC Int. 2019, 102, 1102-1111. [CrossRef] [PubMed]

18. Zhang, S.S.; Liu, Q.T.; Luo, H.L.; Chen, P.; Wu, X.R.; Yang, M.H.; Kong, W.J.; Guo, W.Y. UFLC-MS/MS analysis of four tanshinone components in Salvia miltiorrhizae after ultrasound-assisted extraction. J. Chromatogr. B Anal. Technol. Biomed. Life Sci. 2016, 1017, 204-210. [CrossRef]

19. Eigenmann, D.E.; Xue, G.; Kim, K.S.; Moses, A.V.; Hamburger, M.; Oufir, M. Comparative study of four immortalized human brain capillary endothelial cell lines, hCMEC/D3, hBMEC, TY10, and BB19, and optimization of culture conditions, for an in vitro blood-brain barrier model for drug permeability studies. Fluids Barriers CNS 2013, 10, 33. [CrossRef]

20. Artursson, P.; Karlsson, J. Correlation between oral drug absorption in humans and apparent drug permeability coefficients in human intestinal epithelial (Caco-2) cells. Biochem. Biophys. Res. Commun. 1991, 175, 880-885. [CrossRef]

21. Smith, D.; Artursson, P.; Avdeef, A.; Di, L.; Ecker, G.F.; Faller, B.; Houston, J.B.; Kansy, M.; Kerns, E.H.; Kramer, S.D.; et al. Passive Lipoidal Diffusion and Carrier-Mediated Cell Uptake Are Both Important Mechanisms of Membrane Permeation in Drug Disposition. Mol. Pharm. 2014, 11, 1727-1738. [CrossRef]

22. Wilhelm, I.; Krizbai, I.A. In vitro models of the blood-brain barrier for the study of drug delivery to the brain. Mol. Pharm. 2014, 11, 1949-1963. [CrossRef]

23. Liew, K.F.; Hanapi, N.A.; Chan, K.L.; Yusof, S.R.; Lee, C.Y. Assessment of the Blood-Brain Barrier Permeability of Potential Neuroprotective Aurones in Parallel Artificial Membrane Permeability Assay and Porcine Brain Endothelial Cell Models. J. Pharm. Sci. 2017, 106, 502-510. [CrossRef] [PubMed]

24. Yang, X.; Yan, J.; Feng, J. Treatment with Tanshinone Iia Suppresses Disruption of the Blood-Brain Barrier and Reduces Expression of Adhesion Molecules and Chemokines in Experimental Autoimmune Encephalomyelitis. Eur. J. Pharm. 2016, 771, 18-28. [CrossRef] [PubMed]

25. Yu, X.Y.; Lin, S.G.; Chen, X.; Zhou, Z.W.; Liang, J.; Duan, W.; Chowbay, B.; Wen, J.Y.; Chan, E.; Cao, J.; et al. Transport of Cryptotanshinone, a Major Active Triterpenoid in Salvia Miltiorrhiza Bunge Widely Used in the Treatment of Stroke and Alzheimer's Disease, across the Blood-Brain Barrier. Curr. Drug Metab. 2007, 4, 365-377. [CrossRef] [PubMed] 